\title{
UNUSUAL SPECTRA OF POLARISED RADIO EMISSION OF ACTIVE REGIONS ON THE SUN
}

\author{
L. V. Yasnov*, T. I. Kaltman ${ }^{\dagger}$, and V. M. Bogod ${ }^{\dagger}$
}

\begin{abstract}
Unusual spectra with a significant decrease in circularly polarised emissions (Stokes parameter $V$ ) in the middle part of the microwave range $(6-12 \mathrm{GHz}$ ), sometimes with a change of the polarization sign, were revealed by observations with the radio telescope RATAN-600 in the microwave range $2-16 \mathrm{GHz}$. Such features can be explained by the presence of a hot region in the solar corona. The magnetic field strength of the hot region and the product of the relative gradient of the magnetic field on its thickness are defined.
\end{abstract}

\section{Introduction}

It is possible to characterise unusual properties of radio source spectra in various ways, for example by narrow-band details [Wilson, 1985; Lang and Wilson, 1987; Bogod et al., 2000], a single change of the polarization sign [Alissandrakis et al., 1999; Vourlidas et al., 1997], and double inversion of the polarization sign within a frequency band [Bogod and Tokhchukova, 2003]. We pointed out a new feature in the spectra of some flare active regions; namely, the presence of a minimum in the Stokes parameter $V$ near the middle of the recorded frequency band. We also used model calculations to derive model parameters that match the presented observations.

\section{Observations}

As an example, Figure 1 shows spectra of the antenna temperatures $T V$ and $T I$ of the active region AR 9077 for July 10th, 2000 for Stokes parameters $V$ and $I$, recorded in the radio telescope RATAN-600 at the range 2 - $16 \mathrm{GHz}$.

The spectrum $T V$ has a minimum at a frequency of about $7 \mathrm{GHz}$ and maxima in the ranges $4-6 \mathrm{GHz}$ and $12-14 \mathrm{GHz}$. The archive of solar observations with RATAN-600

\footnotetext{
* Saint-Petersburg State University, Saint-Petersburg 199034, Russia

† Special Astrophysical Observatory, Russian Academy of Sciences, Nizhnij Arkhyz, Russia
} 

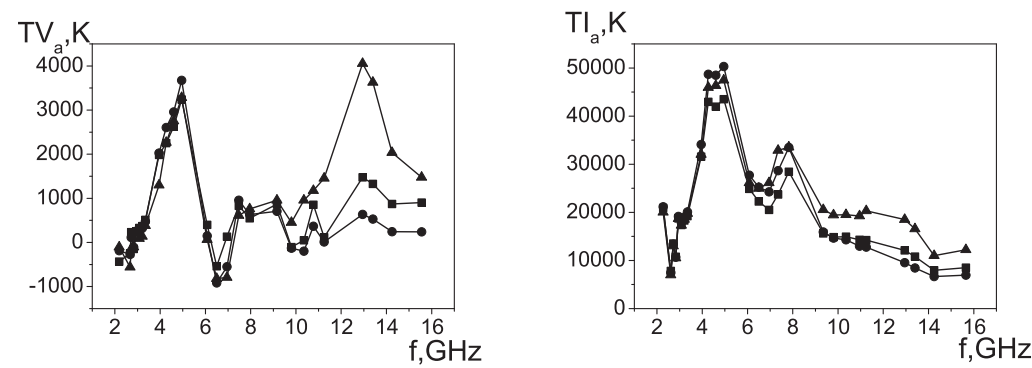

Figure 1: Spectra of the antenna temperatures for the Stokes parameters $V$ (left) and I (right) for AR 9077 on July, 10th, 2000 in its different parts.

contains numerous flare active regions with the following peculiarities of their polarizedflux spectra (Stokes parameter $V$ ):

a) a decrease of the flux in the middle $(6-12 \mathrm{GHz})$ of the recorded frequency band $(2-16$ $\mathrm{GHz}$ ) up to a reversal of the sign of the polarised emission and a corresponding formation of two maxima at the extremities of this range;

b) a decrease of the flux at frequencies where the flux of unpolarized emission in the high frequency range decreases;

c) a sharp decrease of the polarized intensity at about $2-4 \mathrm{GHz}$, accompanied by a shallow reversal of the polarization sign in some cases.

\section{Model}

Let us analyse the above peculiarities of the radio emission of some active regions using model calculations. We will consider a hot loop which is surrounded by the background atmosphere of the active region. As in Zlotnik et al [2007a, 2007b], Brosius and Holman [1987] we chose the torus model as the simplest approximation to the hot loop. The proposed model is thus a standard model of the atmosphere above the sunspot plus a hot coronal loop, approximated as a torus. We carried out calculations only for the central parts of the model as it is quite unrealistic at large distances from the central line.

The magnetic field in the plane $y h$ decreases with distance from the point $y=0$ as $B=\frac{B_{0} R_{0}}{\sqrt{h^{2}+y^{2}}}$, where $y$ is the distance on the photosphere, $h$ - the height, $R_{0}$ - the loop radius ( distance from the point $y=0$ to the loop axis), and $B_{0}$ - the magnetic field on the torus axis. The advantages and drawbacks of such a magnetic-field model are discussed in [Zlotnik et al., 2007a, 2007b], and we will not dwell on them here. Compared to the cited papers, where the emission of coronal loops was studied at decimeter wavelengths, we have increased the magnetic field on the loop axis to 500-700 G, so that the contribution of cyclotron emission from the loop at the second-fourth harmonics are at $4-8 \mathrm{GHz}$, providing a minimum in the spectrum of the polarized emission at 6-10 GHz. The distribution of kinetic temperature was specified as follows: 


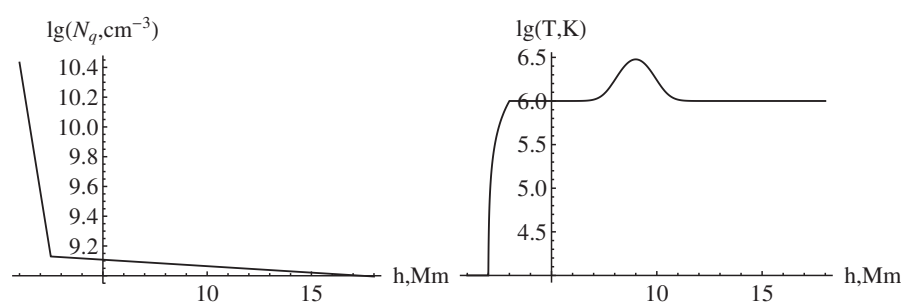

Figure 2: Model of the atmosphere of an active region hosting a hot loop with $R_{0}=9 \cdot 10^{8} \mathrm{~cm}$, $a=10^{8} \mathrm{~cm}, N_{0}=2 \cdot 10^{11} \mathrm{~cm}^{-3}$ for $y=0$.

$$
T=T_{0}\left(\frac{T_{a}}{T_{0}} e^{-\frac{\left(\mathrm{Ro}-\sqrt{h^{2}+y^{2}}\right)^{2}}{a^{2}}}+1\right) .
$$

Here, $T_{0}$ denotes the temperature of the background coronal plasma outside the loop:

$$
T_{0}(h)= \begin{cases}T_{\mathrm{ch}}, & h<h_{\mathrm{ch}} \\ \frac{T_{c}\left(h-h_{\mathrm{ch}}\right)+\left(h_{c}-h\right) T_{\mathrm{ch}}}{h_{c}-h_{\mathrm{ch}}}, & h_{\mathrm{ch}} \leq h \leq h_{c} \\ T_{c}, & h>h_{c},\end{cases}
$$

where $h_{\mathrm{ch}}=2 \cdot 10^{8} \mathrm{~cm}$ and $h_{c}=3 \cdot 10^{8} \mathrm{~cm}$ represent the conventional boundaries of the chromosphere and corona, respectively; between them, the temperature grows from chromospheric values $T_{\mathrm{ch}}=10^{4} \mathrm{~K}$ to coronal values $T_{c}=10^{6} \mathrm{~K}$. The parameter $a$ defines the thickness of the loop. The temperature on the loop axis is determined by $T_{a}$.

The height variation of the electron density was assumed to follow a barometric law [Priest, 1982], taking into account the difference of the temperatures in the chromosphere and corona:

$$
\mathrm{N}_{q}(h)= \begin{cases}\mathrm{N}_{0} e^{-\frac{h}{5000 T_{\mathrm{ch}}}}, & h \leq h_{a} \\ \mathrm{~N}_{0} e^{-\frac{h h_{a}}{5000 T_{c}}-\frac{h_{a}}{5000 T_{\mathrm{ch}}}}, & h>h_{a},\end{cases}
$$

where $h_{a}=\frac{h_{\mathrm{ch}}+h_{c}}{2}$. The adopted value of $\mathrm{N}_{0}$ (about $2 \cdot 10^{11} \mathrm{~cm}^{-3}$ ) yields reasonable densities at the levels of the upper chromosphere, the corona, and the loop. We assumed that the loop was located at the solar-disk centre and the line of sight was parallel to the $h$ axis. Figure 2 shows the height distributions of the electron temperature, density in the adopted model for $\mathrm{y}=0, R_{0}=9 \cdot 10^{8} \mathrm{~cm}, a=10^{8} \mathrm{~cm}, T_{a}=2 \cdot 10^{6} \mathrm{~K}$, and $N_{0}=2 \cdot 10^{11} \mathrm{~cm}^{-3}$.

\section{Model Calculations}

Thermal cyclotron emission is calculated according to the well-known formulae [Zheleznyakov, 1997] taking into account harmonics from 2 to 5 . The spectra of the brightness temperatures of Stokes parameters $V$ and $I$ ( $T V$ and $T I$ ) near the loop centre 
$\left(y=2 \cdot 10^{8} \mathrm{~cm}\right)$ for $R_{0}=9 \cdot 10^{8} \mathrm{~cm} ; a=10^{8} \mathrm{~cm} ; N_{0}=2 \cdot 10^{11} \mathrm{~cm}^{-3}$ and $B_{0}=500 \mathrm{G}$ and $700 \mathrm{G}$ are shown in Figure 3.

The magnetic field strength in the loop must be about 300-700 G in order to match the majority of the observed spectra. For active regions, where the minimum in the spectrum is close to $10 \mathrm{GHz}$, the magnetic field strength must be about $700 \mathrm{G}$.

If the loop width is doubled by setting $a=2 \cdot 10^{8} \mathrm{~cm}$, the width of the low frequency maximum increases (Figure 4). This enables us to estimate the loop thickness from the width of the maximum (see section 5).

Notice that model calculations yield the position of the minimum of the polarization $(T V)$ at frequencies where the intensity $(T I)$ decreases in the high frequency range. Thus, even such an imperfect model of an active region qualitatively matches the peculiarities of the observed spectra mentioned earlier.

Calculations of the spectra with other assumptions about the dependence of magnetic field on height [Takakura, 1972] have been carried out. The spectra for the different distributions of the magnetic field with identical strength in a loop were similar: there is a minimum of polarization between the maxima at the same frequencies. The increase in electronic density in a loop compared to the background atmosphere practically does not change the spectra TV and TI, leaving all features at the same frequencies. But a temperature decrease in a loop below a temperature of the background atmosphere radically changes the spectra TV and TI. This means that a basic feature of a model which can give the observable spectra is the high temperature in a loop in comparison with the temperature of the background atmosphere.

Table 1: Estimations of the magnetic field strength and the magnetic field gradient

\begin{tabular}{ccccc}
\hline \hline AR, & $f_{\text {max }}$, & $\Delta f$, & $B_{0}, G$ & $\frac{B^{\prime}}{B} \Delta h$ \\
date & $G H z$ & $G H z$ & $G H z$ & \\
\hline 9077 & 4.9 & 1.7 & 580 & 0.35 \\
2000.07 .10 & & & & \\
\hline 9077 & 4.0 & 2.1 & 480 & 0.48 \\
2000.07 .16 & & & & \\
\hline 10488 & 4.9 & 3.1 & 600 & 0.63 \\
2003.10 .29 & & & & \\
\hline 10570 & 5.0 & 2.4 & 600 & 0.48 \\
2004.03 .05 & & & & \\
\hline 10570 & 4.6 & 1.2 & 550 & 0.26 \\
2004.03 .06 & & & & \\
\hline 10570 & 5.0 & 2.9 & 450 & 0.58 \\
2004.03 .07 & & & & \\
\hline \hline
\end{tabular}



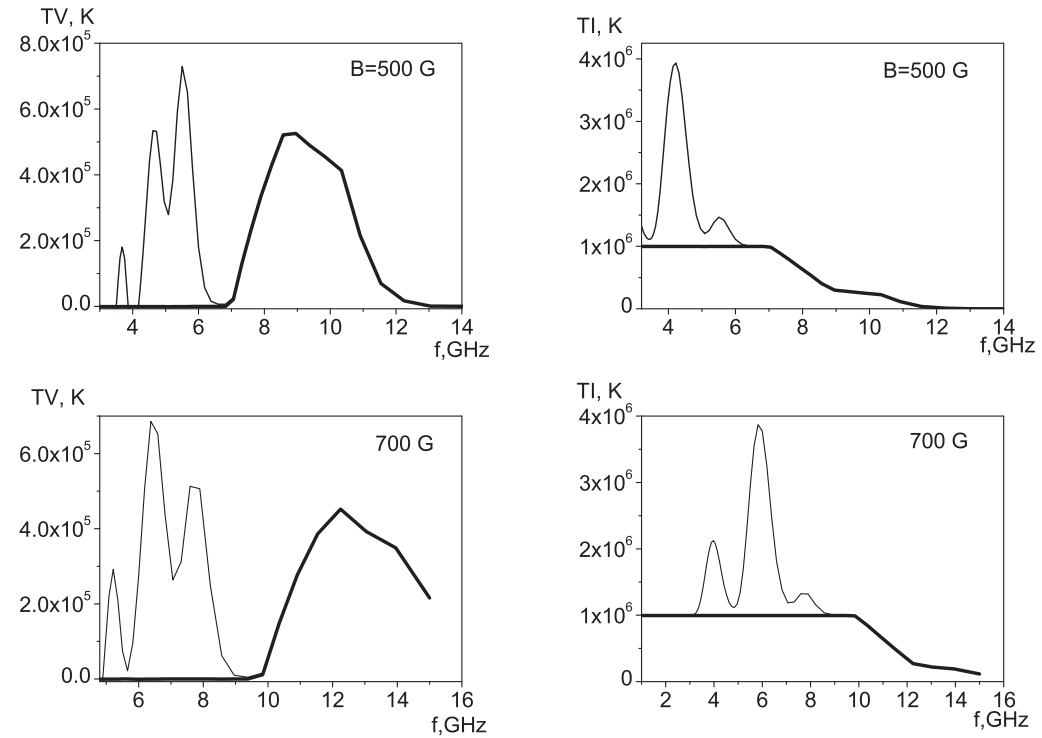

Figure 3: Spectra of the brightness temperatures $T V$ and $T I$ (Stokes parameters $V$ and I) near the loop centre. $B_{0}=500 G$ (the top row) and $B_{0}=700 G$ (the bottom row). The solid curve shows the emission spectra for a source without a loop.
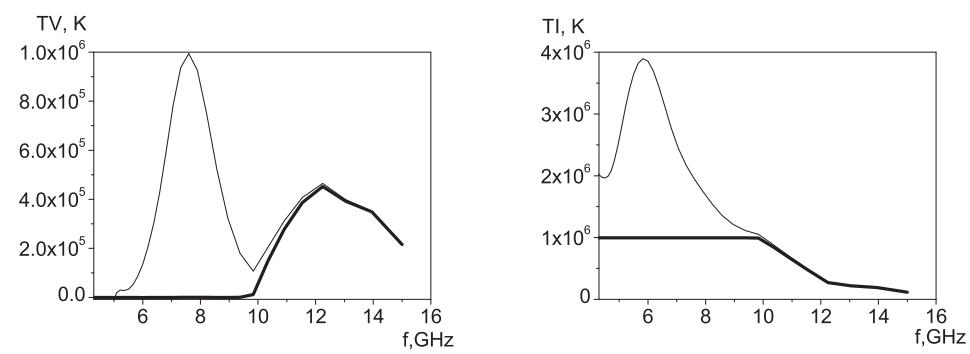

Figure 4: Spectra of the brightness temperatures $T V$ and $T I$ (Stokes parameters $V$ and I) for $a=2 \cdot 10^{8} \mathrm{~cm}, B_{0}=700 \mathrm{G}$.

\section{Estimations of Physical Conditions in a Loop}

Our calculations enable us to note some relationships between the model parameters and the parameters of the observed spectrum $T V$, and to obtain some estimates of physical conditions in a loop. Assuming that the low-frequency maximum in $T V$ is related to the emission mainly at the third harmonic of the gyrofrequency, we find (e.g., from Figure 1) from the frequency of the low-frequency maximum $\left(f_{\max }=4.9 \cdot 10^{9} \mathrm{~Hz}\right)$ that $B_{0}=580$ $\mathrm{G}$, which compares well to the accepted value $(500 \mathrm{G})$. This enables us to determine the 
magnetic field in hot regions of the active regions under study (Table). The width of the low-frequency maximum $\Delta f$ depends on the loop width $\Delta h$ and the magnetic field gradient in the loop $B^{\prime}: \frac{\Delta f}{f_{\max }}=\frac{B^{\prime}}{B} \Delta h$. The results of the corresponding calculations are also listed in the Table.

\section{Conclusions}

Based on the spectral polarization observations of the Sun with the RATAN-600 radio telescope, we have found the following spectral features in a number of the microwave sources in active regions:

a) a minimum of the polarised emission at intermediate frequencies in the recorded band, up to a reversal of the polarization sign and the corresponding formation of two maxima at the extremities of the frequency range;

b) a minimum of the polarized emission at frequencies where the unpolarised emission in the high-frequency part of the range falls;

c) a sharp decrease in the intensity of the polarized emission around $2-4 \mathrm{GHz}$, with in some cases a change in the sign of the polarization.

We have interpreted these features using a model with a hot coronal magnetic loop. Our calculations indicate a good qualitative agreement between the calculated and observed spectra of polarised and unpolarised emission. Details of the obtained spectra demonstrate how it is possible to diagnose the physical conditions in a coronal loop using information about the spectrum and the polarization of the microwave emission of a source. We have obtained the following parameters of hot regions in the emission sources for a number of active regions: the magnetic field $(360-450 \mathrm{G})$ and the product of the magnetic field gradient and the size of the region $(0.26-0.63)$. Details of the model of the magnetic field have little effect on these characteristics, due to the small transverse size of the loop. The basic feature of a model which can reproduce the observable spectra is the high temperature in a loop in comparison with the temperature of the background atmosphere.

Acknowledgements. This study is partially supported by the Russian Academy of Sciences program OFN-16 and PAN-16 and by the Russian Foundation for Basic Research 08-02-00378 and 09-02-00111.

\section{References}

Alissandrakis, C., N. Gizani, V. Bogod, V. Garaimov, G. Gelfreikh, V. Zheleznyakov, and E. Zlotnik, RATAN-600 Observations of unusual inversion of polarization in microwave sources, The 9th European Meeting on Solar Physics, edited by A. Wilson, ESA SP-448, 1225-1229, 1999.

Bogod, V. M., and S. Kh. Tokhchukova, Peculiarities of the microwave emission from active regions generating intense solar flares, Astron. Lett., 29, 263-273, 2003. 
Bogod, V.M., V.I. Garaimov, V.V. Zheleznyakov, and E. Ya. Zlotnik, Detection of a cyclotron line in the radio spectrum of a solar active region and its interpretation, Astron. Rep., 44, 271-277, 2000.

Brosius, J., and G. Holman, Theoretical models of free-free microwave emission from solar magnetic loops, Astrophys. J., 327, 417-426, 1988.

Lang, K. R., R.F. Wilson, K. L. Smith, and K. T. Strong, Solar active region physical parameters inferred from a thermal cyclotron line and soft X-ray spectral lines, Astrophys. J., 322, 1044, 1987.

Priest, E. R., Solar magnetohydrodynamics, Dordrecht, D. Reidel Publ. Company, 1982.

Takakura, T., The self absorption of gyro-synchrotron emission in a magnetic dipole field: microwave impulsive burst and hard X-ray burst, Solar Phys., 26, 151-175, 1972.

Vourlidas, A., T.S. Bastian, and M. J. Aschwanden, The structure of the solar corona above sunspots as inferred from radio, X-ray, and magnetic field observations, $A_{s-}$ trophys. J., 489, 403-425, 1997.

Willson, R.F., VLA observation of solar active regions at closely spaced frequencies: evidence for thermal cyclotron line emisson, Astrophys. J., 298, 911-917, 1985.

Zheleznyakov, V. V., Radio emission of the sun and planets, edited by J. S. Hey, Pergamon, Oxford, 1970.

Zlotnik, E. Ya., T. I. Kaltman, and J. A. Sheiner, Thermal cyclotron radiation from hot coronal loops and peculiarities of the polarization structure of solar microwave emission sources: I. Brightness temperature, Astron. Lett., 33, 168-181, 2007.

Zlotnik, E. Ya., T. I. Kaltman, and J. A. Sheiner, Thermal cyclotron radiation from hot coronal loops and peculiarities of the polarization structure of solar microwave emission sources: II. Integrated characteristics, Astron. Lett., 33, 327-339, 2007. 
